\title{
Comportamento de leitões na fase de creche submetidos ao enriquecimento ambiental
}

\author{
Behavior of piglets in the nursery phase subjected to environmental enrichment
}

\author{
Tiago Vieira de Andrade ${ }^{1}$, Larissa Siqueira Andrade ${ }^{2}$, Leonardo Atta Farias ${ }^{2}$, Paulo Henrique Amaral \\ Araújo de Sousa ${ }^{3^{*}}$, Laylson da Silva Borges ${ }^{4}$, Raimundo Nonato Vieira Santos ${ }^{2}$, Barbara Silveira \\ Leandro de Lima ${ }^{5}$, Cicero Pereira Barros Junior ${ }^{3}$ e Sandro Tavares Carvalhinho ${ }^{2}$
}

\author{
${ }^{1}$ Universidade Federal de Goiás, Goiânia, GO, Brasil. \\ ${ }^{2}$ Universidade Federal do Piauí, Bom Jesus, PI, Brasil. \\ ${ }^{3}$ Universidade Estadual do Oeste do Paraná, Marechal Cândido Rondon, PR, Brasil. *Autor para correspondência: \\ paullo_ap1@hotmail.com \\ ${ }^{4}$ Universidade Federal do Piauí, Teresina, PI, Brasil. \\ ${ }^{5}$ Universidade Federal do Ceará, Fortaleza, CE, Brasil
}

Submissão: 22/09/2016 / Aceite: 05/04/2019

\begin{abstract}
RESUMO
O enriquecimento ambiental constitui no aperfeiçoamento das instalações na forma de estímulos ambientais necessários para alcançar o bem-estar dos animais nas condições psíquico, fisiológico e melhor qualidade de vida. Nos últimos anos, o bem-estar animal vem se tornando um dos maiores desafios para os modernos sistemas de produção. A pressão da sociedade e do mercado internacional para que os sistemas de produção respeitem o bem-estar animal, aumenta a necessidade de pesquisas que possam gerar conhecimentos sobre possíveis formas de se produzir suínos garantindo a lucratividade do sistema em combinação com a manutenção da qualidade de vida dos mesmos. Sendo assim, objetivou-se com este estudo avaliar o comportamento de leitões na fase de creche submetidos a enriquecimento ambiental. Foram utilizados 12 suínos na fase de creche, distribuídos em dois tratamentos, com presença e ausência de enriquecimento ambiental. Para avaliação das variáveis comportamentais foram observadas as seguintes características: em pé, deitado, explorando, cheirando, bebendo, comendo, ócio, fuçando, defecando, urinando, sentado, andando, vocalizando, mordendo, brincando e brigando. As observações foram realizadas a cada 10 minutos durante seis dias. Houve diferença significativa $(p<0,05)$ para os comportamentos brincando, comendo e mordendo em relação aos tratamentos avaliados. Não houve diferença significativa $(p>0,05)$ para o comportamento brigando em relação aos tratamentos, dias e animais. O comportamento dos animais foi influenciado pela presença do enriquecimento ambiental, em que o mesmo proporcionou a diminuição de alguns comportamentos indesejáveis nos sistemas de produção.
\end{abstract}

PALAVRAS-CHAVE: bem-estar animal, comportamento exploratório, suinocultura.

\begin{abstract}
Environmental enrichment consists of the enhancement of facilities by means of the necessary environmental stimuli to achieve animal welfare in psychical, physiological, and to improve quality of life conditions. In recent years welfare has been turning into one of the biggest challenges for modern production systems. There is a great pressure from civil society and international market claiming for production systems to respect animal welfare. This increases the need for studies that generate knowledge on how to raise pigs in order to ensure the profitability of the system and the maintenance of the quality of life for these animals. Therefore, the aim of this study was to evaluate the behavior of piglets in nursery phase subjected to environmental enrichment. A total of 12 pigs in the nursery phase were distributed into two treatments (with and without environmental enrichment). The following behaviors were evaluated: standing, laying, exploring, smelling, drinking, eating, idling, digging, defecating, urinating, sitting, walking, vocalizing, biting, playing, and fighting. Piglet behavior was recorded every 10 minutes during six days. Significant differences $(p<0.05)$ between treatments were found for playing, eating and biting. No significant differences ( $p>0.05$ ) were found for fighting behavior regarding the treatments, days and animals. Animal behavior was affected by the presence of environmental enrichment, which decreased some undesirable behaviors in production systems.
\end{abstract}




\section{INTRODUÇÃO}

O desmame dos leitões ocorre, normalmente, aos 21 dias de idade quando se inicia a fase de creche que é um dos períodos mais estressantes para os animais pela separação da mãe, interrupção da lactação, mistura com animais desconhecidos e alteração inesperada na alimentação e ambiente, o que implica em disputas por competição e hierarquia (ANDRADE et al. 2015).

O estudo do comportamento de leitões na fase inicial de produção é originado da preocupação em compreender os hábitos dos animais e da sua acomodação às condições do ambiente, visando cada vez mais estabelecer qual medida influencia o desempenho animal, de acordo com a função adaptativa da linhagem, sabendo que os animais são seres sociáveis e sensitivos (FOPPA et al. 2014).

O estudo do comportamento dos animais é motivado por necessidades básicas dos mesmos, seja para buscar alimento, água, abrigo ou pela simples necessidade exploratória dos suínos (REGO NETO et al. 2013). Diante do contexto, este assunto assume papel preponderante na produção animal, uma vez que para racionalizar os métodos de produção é necessário desenvolver técnicas de manejo, alimentação e instalações que interferem e dependem do comportamento dos animais. Assim, o estudo do comportamento animal pode otimizar a criação animal, principalmente em sistemas intensivos de produção convencional (VASCONCELOS et al. 2015).

O enriquecimento ambiental é um método efetivo no manejo animal que procura ampliar a qualidade de vida em confinamento, visando melhorar o bem-estar psicológico e fisiológico dos animais, por meio de modificações ambientais. Sendo assim, este método consiste em proporcionar melhores condições de vida aos animais, culminando em melhor desempenho produtivo (CAMPOS et al. 2010).

Os suínos são considerados animais extremamente curiosos e inteligentes, apresentando um complexo repertório comportamental. Para eles, a monotonia dos ambientes físicos estéreis, presente nos sistemas atuais de produção, se traduz em maior período de inatividade, contrário ao comportamento exploratório dessa espécie (LUZ et al. 2017).

Para reduzir os fatores estressantes, tem-se proposto à introdução de práticas de enriquecimento ambiental para suínos na fase inicial de produção, de modo a tornar o ambiente mais adequado às suas necessidades comportamentais. PINHEIRO et al. (2009) descreveram que a utilização de objetos nas baias como forma de brinquedos são algumas alternativas para leitões, como proposta à diminuição da inatividade e estímulo à demonstração do comportamento natural, proporcionando assim maior bem-estar e melhor exploração do seu potencial produtivo. Nesse contexto, objetivou-se com essa pesquisa avaliar o comportamento de leitões na fase de creche submetidos à presença de enriquecimento ambiental.

\section{MATERIAL E MÉTODOS}

O experimento foi realizado no período de 29 de maio a 03 de junho de 2012, no setor de suinocultura do Colégio Técnico de Bom Jesus, localizado próximo ao campus Professora Cinobelina Elvas, da

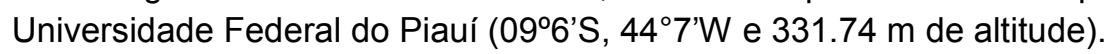

A região apresenta condições climáticas do tipo semiárido, que significa clima seco e quente com estação chuvosa no verão, atrasando-se para o outono, comuns a todas as regiões do Nordeste brasileiro (SILVA et al. 2015, SOUSA et al. 2016). A umidade relativa e a temperatura média do ar diária foram registradas pela estação meteorológica da Universidade Federal do Piauí, Bom Jesus, PI (Figura 1). Foram utilizados 12 suínos em fase de creche com peso vivo médio de $24,5 \mathrm{~kg}$, distribuídos em um delineamento inteiramente casualizado com dois tratamentos e seis repetições por tratamento.

O tratamento I foi composto por seis suínos da linhagem Agroceres PIC, sendo três machos castrados e três fêmeas, que receberam o enriquecimento ambiental em forma de brinquedo, sendo instaladas três correntes que ficavam suspensas com uma altura média de $18 \mathrm{~cm}$ do piso. O tratamento II foi composto por seis suínos da linhagem Agroceres PIC, sendo três machos castrados e três fêmeas que não receberam o enriquecimento ambiental.

O galpão onde foram realizadas as coletas é construído em alvenaria e cimento, com 3 metros de pé direito, possuindo cobertura de duas águas com telha de cerâmica no sentido leste/oeste. Os animais estavam alojados em baias, com dimensões de 4,0 m x 3,5 m, com piso de cimento grosso havendo a declividade para o escoamento dos dejetos.

Os suínos machos foram castrados aos 15 dias de idade na fase de lactação, totalizando 12 leitões na fase de creche em baias, com comedouro de cano PVC de $300 \mathrm{~mm}$ fixado ao piso e bebedouro tipo 
chupeta. A constituição bromatológica da ração fornecida aos animais está apresentada na Tabela 1.

$O$ arraçoamento dos animais foi realizado no comedouro da baia dentro do galpão, em média $750 \mathrm{~g} / \mathrm{animal} / \mathrm{dia}$, sendo essa quantidade dividida em dois fornecimentos diários, um às $8 \mathrm{~h}$ e outro às $16 \mathrm{~h}$.

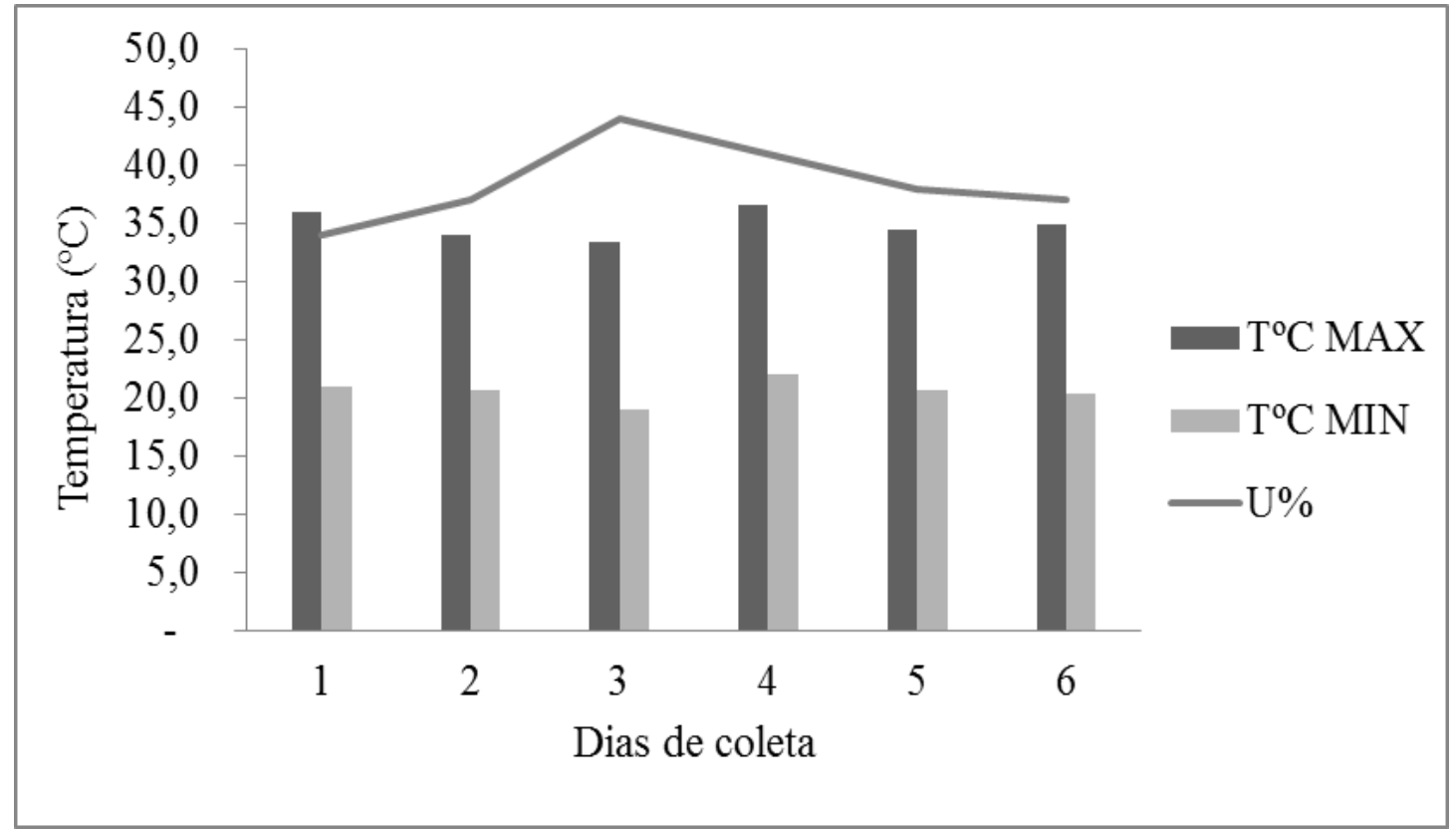

Fonte: Arquivo pessoal (2016).

Figura 1. Médias das variáveis ambientais, temperatura mínima ( $\left.T^{\circ} \mathrm{C} M I N\right)$ e máxima ( $\left.{ }^{\circ} \mathrm{C} M A X\right)$, e umidade relative do ar (U\%) durante o período experimental.

Figure 1. Means of environmental variables, minimum ( $T^{\circ} \mathrm{C}$ MIN) and maximum ( $\left.T^{\circ} \mathrm{C} M A X\right)$ temperature, and relative humidity $(\mathrm{AH} \%)$ during the experimental period.

Os animais foram avaliados durante seis dias consecutivos, das $7 \mathrm{~h}$ às $17 \mathrm{~h}$. Foram registrados os dados referentes às atividades comportamentais de cada animal e anotados em planilhas próprias (etograma) por dois observadores treinados, mantidos em sistema de revezamento e posicionados de modo a não interferir no comportamento natural dos animais, evitando movimentos bruscos que pudessem alterar a situação existente do ambiente.

Tabela 1. Composição nutricional das rações fornecidas para os suínos na fase de creche.

Table 1. Nutritional composition of rations supplied to piglets in the nursery phase.

\begin{tabular}{cc}
\hline Composição Nutricional (\%) & Fase Inicial \\
\hline Umidade (Max.) & 13 \\
Proteína & 18 \\
Extrato Etéreo & 4 \\
Matéria Fibrosa & 5 \\
Matéria Mineral & 7 \\
Cálcio & 1,2 \\
Fósforo & 0,7 \\
\hline
\end{tabular}

Fonte: Arquivo pessoal (2016).

As observações comportamentais foram realizadas da forma direta, com determinação instantânea, utilizando intervalo amostral de 10 minutos por observação durante $9 \mathrm{~h} /$ dia em seis dias, totalizando 3.960 observações.

As variáveis comportamentais observadas foram quesitos de etograma adaptado por KIEFER et al. (2010), sendo elas: em pé, deitado, explorando, cheirando, bebendo, comendo, ócio, fuçando, defecando, urinando, sentado, andando, vocalizando, mordendo, brincando e brigando.

Os dados coletados das variáveis comportamentais foram submetidos à análise de variância utilizando-se o pacote estatístico SAS, versão 9.3 (SAS Institute 2003) e para a comparação das médias entre os tratamentos foi utilizado o teste do $X^{2}$ (Qui-Quadrado) a $5 \%$ de probabilidade. 


\section{RESULTADOS E DISCUSSÃO}

Os comportamentos dos suínos estão apresentados na Tabela 2. Foi observado que a característica brincando apresentou diferença significativa $(p<0,05)$ em relação aos tratamentos estudados, demonstrando assim que a presença de enriquecimento em forma de correntes influenciou o comportamento dos animais criados em sistema intensivo.

Tabela 2. Teste de significância para as variáveis comportamentais de suínos na fase de creche, mantidos com presença e ausência de enriquecimento ambiental.

Table 2. Significance test for behavioral variables of piglets in the nursery phase kept with and without environmental enrichment.

\begin{tabular}{lllcc}
\hline Características & Horas & Animais & Dia & Tratamento \\
\hline Em pé & $0,0001^{*}$ & $0,0006^{*}$ & $0,757^{\text {ns }}$ & $0,02^{*}$ \\
Deitado & $0,0001^{*}$ & $0,0001^{*}$ & $0,598^{\text {ns }}$ & $0,0001^{*}$ \\
Explorando & $0,0001^{*}$ & $0,0011^{*}$ & $0,011^{\text {ns }}$ & $0,0001^{*}$ \\
Cheirando & $0,574^{\text {ns }}$ & $0,08^{\text {ns }}$ & $0,008^{\text {ns }}$ \\
Bebendo & $0,086^{\text {ns }}$ & $0,013^{\text {ns }}$ & $0,257^{\text {ns }}$ \\
Comendo & $0,0001^{*}$ & $0,074^{\text {ns }}$ & $0,460^{\text {ns }}$ & $0,969^{\text {ns }}$ \\
Ócio & $0,0001^{*}$ & $0,0001^{*}$ & $0,0001^{*}$ & $0,001^{\text {ns }}$ \\
Fuçando & $0,0001^{*}$ & $0,909^{\text {ns }}$ & $0,050^{\text {ns }}$ & $0,107^{\text {ns }}$ \\
Defecando & $0,059^{*}$ & $0,698^{\text {ns }}$ & $0,124^{\text {ns }}$ & $0,795^{\text {ns }}$ \\
Urinando & $0,441^{\text {ns }}$ & $0,882^{\text {ns }}$ & $0,6^{\text {ns }}$ & $1,00^{\text {ns }}$ \\
Sentado & $0,723^{\text {ns }}$ & $0,0001^{*}$ & $0,004^{*}$ & $0,0001^{*}$ \\
Andando & $0,550^{\text {ns }}$ & $0,0005^{*}$ & $0,0004^{*}$ & $0,0001^{*}$ \\
Vocalizando & $0,0001^{*}$ & $0,0001^{*}$ & $0,0001^{*}$ & $0,0001^{*}$ \\
Mordendo & $0,0001^{*}$ & $0,0001^{*}$ & $0,495^{\text {ns }}$ & $0,0001^{*}$ \\
Brincando & $0,884^{\text {ns }}$ & $0,0001^{*}$ & $0,85^{\text {ns }}$ & $0,0001^{*}$ \\
Brigando & $0,147^{\text {ns }}$ & $0,528^{\text {ns }}$ & $0,528^{\text {ns }}$ & $0,008^{\text {ns }}$ \\
\hline
\end{tabular}

${ }^{*}$ Significativo $(p<0,05) ; n s=$ não significativo. Fonte: Arquivo pessoal (2016).

Ao avaliaram o desempenho de suínos na fase de creche, com presença e ausência de enriquecimento ambiental, CAMPOS et al. (2010) observaram que os animais que receberam o tratamento com presença de enriquecimento ambiental manifestaram mais as características comportamentais da sua espécie, em relação aos animais que receberam o tratamento sem enriquecimento ambiental.

A característica comendo apresentou diferença significativa $(p<0,05)$ em relação às horas. Entretanto, não foi observada diferença significativa em relação aos animais, dia e tratamento. Esse fato pode ser explicado por conta dos animais terem um horário fixo do dia para maior consumo de ração que se apresenta entre $8 \mathrm{~h}$ até $16 \mathrm{~h} 30 \mathrm{~min}$ atendendo desse modo as suas exigências nutricionais.

Ao avaliarem o comportamento de suínos em confinamento na fase de creche, REGO NETO et al. (2013) observaram que a característica comendo não apresentou diferença significativa quando relacionada dentro de horas, dias e animais, indicando que os leitões se alimentam todos os dias na mesma hora, corroborando com o resultado encontrado neste estudo.

Houve diferença significativa $(p<0,05)$ entre os tratamentos e os animais para o comportamento mordendo, constatando que o enriquecimento ambiental influenciou positivamente em um comportamento que é indesejável no sistema de produção (Figura 2), pois provoca escoriações e possíveis inflamações nas áreas que são mordidas. VAN DE WEERD et al. (2006) introduziram diferentes objetos de enriquecimento ambiental e observaram que houve redução $(p<0,05)$ de mordidas na cauda entre suínos na fase de crescimento, mostrando melhora no bem-estar dos animais.

A variável brigando não apresentou diferença significativa entre os tratamentos, dias e animais. Esse fato pode ser justificado pela quantidade satisfatória de artefatos (brinquedos) atraentes fornecidos aos animais, denotando a grande importância do enriquecimento ambiental para melhor bem-estar dos suínos na fase de creche. No entanto, foi observado diferença significativa $(p<0,05)$ para as horas estudadas, provavelmente pelo clima que se mostrou estressante para os animais nos horários mais quentes do dia.

MIRANDA et al. (2012) relataram que com a elevação gradativa da temperatura, os leitões apresentaram comportamento agitado, seguido de brigas, disputa pelo espaço próximo ao bebedouro, 
inquietação, ocorrendo principalmente na faixa de temperatura de 23 a $30^{\circ} \mathrm{C}$.

Pode-se observar na Figura 2 as frequências das variáveis comportamentais dos suínos na fase de creche avaliados com a presença e ausência do enriquecimento ambiental. Nota-se que as características em pé, explorando, sentado, mordendo e brincando apresentaram frequências superiores para o tratamento com enriquecimento ambiental. Entretanto, para o tratamento sem enriquecimento ambiental os suínos apresentaram frequências maiores para as características deitado, andando e vocalizando.

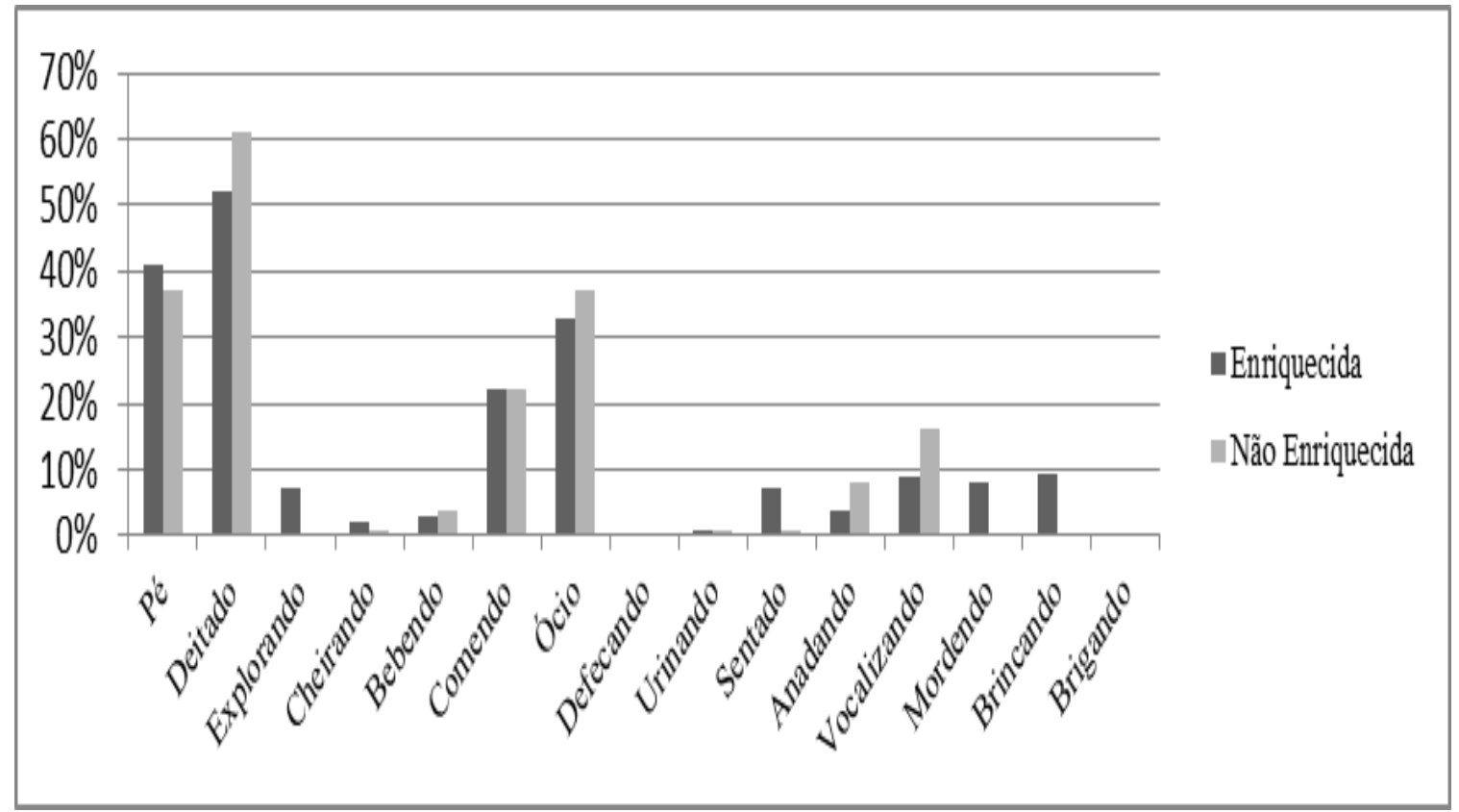

Fonte: Arquivo pessoal (2016).

Figura 2. Frequências das variáveis comportamentais dos suínos na fase de creche, mantidos com presença e ausência de enriquecimento ambiental.

Figure 2. Frequency of behavioral variables of piglets in the nursery phase kept with and without environmental enrichment.

Estudo que comprova que o enriquecimento ambiental promove alterações comportamentais e podem contribuir como instrumento para avaliar o bem-estar dos suínos foram realizados por VASCONCELOS et al. (2015) que adotaram uma corrente como brinquedo para enriquecer o ambiente de animais na fase de crescimento; os autores supracitados observaram que houve alteração nas frequências de algumas variáveis comportamentais, dentre elas as variáveis deitado, chafurdando, comendo, brincando e brigando.

As características cheirando, bebendo, comendo, ócio, fuçando, defecando, urinando e brigando apresentaram frequências semelhantes para os tratamentos avaliados. Destaca-se que os leitões na fase de creche estão se adequando aos manejos e a rotina diária de um confinamento, provavelmente, por isso alguns comportamentos não apresentaram diferenças significativas em relação aos tratamentos estudados (LUZ et al. 2017). VASCONCELOS et al. (2015), ao avaliarem o comportamento de suínos na fase de crescimento criados em ambiente enriquecido, não observaram diferença significativa para os comportamentos bebendo, ócio, defecando, sentado, andando e vocalizando, mostrando assim que os animais estudados apresentaram frequências iguais para esses comportamentos, corroborando com os valores encontrados nesse estudo. Resultados semelhantes foram encontrados por MOI et al. (2016) ao avaliarem o comportamento de leitões desmamados submetidos à ambiente enriquecido com a utilização de feno.

De encontro a isso, $\mathrm{RICCl}$ et al. (2012), trabalhando com matrizes, observaram que o enriquecimento ambiental influenciou significativamente os comportamentos alimentação e consumo de água. Os autores encontraram frequências de $72,53 \%$ e $27,47 \%$ para o comportamento alimentação e $72,28 \%$ e $27,72 \%$ para o comportamento consumo de água com presença e ausência de ambiente enriquecido, nessa mesma ordem.

MACHADO et al. (2017) destacaram que, atualmente, o desafio da comunidade científica é desenvolver técnicas de enriquecimento ambiental que atinjam os objetivos de melhorar o bem-estar dos animais ao mesmo tempo em que são facilmente implementados e economicamente viáveis. Para 
NANNONI et al. (2017), o enriquecimento ambiental é capaz de melhorar o funcionamento biológico dos suínos confinados. Ainda, segundo esses autores, um enriquecimento bem aplicado deve, portanto, diminuir a incidência de padrões anormais do comportamento e aumentar a frequência de comportamentos específicos da espécie.

\section{CONCLUSÃO}

O comportamento dos suínos foi influenciado pela presença do enriquecimento ambiental, que proporcionou a diminuição de alguns comportamentos indesejáveis para os sistemas de produção da espécie.

\section{REFERÊNCIAS}

ANDRADE TV et al. 2015. Aspectos relacionados ao bem-estar animal na produção de suínos. Journal of Animal Behaviour Biometeorology 3: 124-127.

CAMPOS JA et al. 2010. Enriquecimento ambiental para leitões na fase de creche advindos de desmame aos 21 e 28 dias. Revista Brasileira de Ciências Agrárias 2: 272-278.

FOPPA L et al. 2014. Enriquecimento Ambiental e Comportamento de Suínos: Revisão. Brazilian Journal of Biosystems Engineering 8: 1-7.

KIEFER C et al. 2010. Respostas de suínos em terminação mantidos em diferentes ambientes térmicos. Revista Brasileira de Saúde e Produção Animal 11: 496-504.

LUZ CSM et al. 2017. Physiological Parameters of Pigs Raised with and without Environmental Enrichment. Journal of Agricultural Science 4: 176-185.

MACHADO SM et al. 2017. Behavior of Pigs Reared in Enriched Environment: Alternatives to Extend Pigs Attention. PLOS ONE 12: 1-18.

MIRANDA KOS et al. 2012. Efeito das condições ambientais no nível de ruído emitido por leitões. Engenharia Agrícola 32: $435-445$.

MOI A et al. 2016. Comportamento de leitões desmamados submetidos a ambiente enriquecido com a utilização de feno. ÁGORA Revista Eletrônica 1: 79-83.

NANNONI E et al. 2017. Enrichments For Pigs: Improving Animal-Environment Relations. Fourth International Conference on Animal-Computer Interaction Proceedings 15: 1-6.

PINHEIRO JV. 2009. A pesquisa com bem estar animal tendo como alicerce o enriquecimento ambiental através da utilização de objeto suspenso no comportamento de leitões desmamados e seu efeito como novidade. Dissertação (Mestrado em Zootecnia). Pirassununga: USP. 80p.

REGO NETO AA et al. 2013. Avaliação do comportamento de suínos em confinamento na fase de creche no Sul do Piaui. Pubvet 7: 619-706.

RICCI GD et al. 2012. Análise do comportamento de matrizes suínas lactantes com o uso de enriquecimento ambiental na maternidade. In: 6a Jornada de Iniciação Científica Embrapa e II Seminário de Pesquisa e Extensão da UnC. Anais... Concórdia: Embrapa. p.167.

SAS Institute. 2003. Statistical Analysis System. User's Guide. Versão 9.1. Cary: SAS Institute Inc. 129p.

SILVA AL et al. 2015. Características termorreguladoras de bovinos mestiços Holandês x Gir no sul do estado do Piauí. Journal of Animal Behaviour and Biometeorology 3: 97-100.

SOUSA PHAA et al. 2016. Feeding behavior of Santa Inês sheep in pastures of Cynodon dactylon and Andropogon gayanus. Journal of Animal Behaviour and Biometeorology 4: 96-100.

VAN DE WEERD HÁ et al. 2006. Effects of species relevant environmental enrichment on the behaviour and productivity of finishing pigs. Applied Animal Behaviour Science 99: 230-247.

VASCONCELOS EKF et al. 2015. Behavior of pigs in the growth phase raised in an enriched environment. Journal of Animal Behaviour and Biometeorology 3: 120-123. 